\title{
Materiales con propiedades tecnológicas obtenidos por modificación química de un caolín natural
}

\author{
C. BELVER, M.A. BAÑARES, M.A. VICENTE \\ Dpto. Química Inorgánica. Fac. Ciencias Químicas. Universidad de Salamanca. 37008 Salamanca.
}

\begin{abstract}
En este trabajo se ha estudiado la síntesis de materiales de alto valor añadido a partir de un caolín natural. Para ello el caolín fue pre-activado mediante calcinación para dar lugar a la formación de metacaolines, sólidos amorfos susceptibles de ser modificados por tratamientos químicos de una manera sencilla y poco costosa. Estos sólidos fueron posteriormente sometidos a tratamientos ácidos y básicos para modificar su estructura y propiedades. La activación ácida bajo unas condiciones específicas dio lugar a la síntesis de sólidos constituidos mayoritariamente por sílice con buenas propiedades superficiales, desarrollándose tanto su superficie externa como interna, revelándose como sólidos que pueden ser empleados como adsorbentes y soportes catalíticos. Por su parte, la modificación causada por la activación básica dio lugar a la cristalización de la zeolita K-F (Z), presentándose como un método sencillo y eficaz para la síntesis de este material.
\end{abstract}

Palabras clave: caolín, activación ácida, activación básica.

\section{Technological materials obtained by chemical modification of kaolin}

The synthesis of materials of high added value from a natural kaolin has been studied. The kaolin was pre-activated by means of calcination to obtain the metakaolins, which are amorphous solids capable of being modified by simple and non expensive chemical treatments. These solids were then submitted to acid and basic treatments to modify their structure and properties. The acid activation under specific conditions gave place to the synthesis of solids mainly constituted by silica with good textural properties, developing so much their external and internal surface, these solids may be used like adsorbentes and catalytic supports. On the other hand, the basic activation produced the modification of the metakaolins and it gave place to the crystallisation of the zeolite K-F (Z), appearing as a simple and effective method for the synthesis of this material.

Keywords: kaolin, acid treatment, alkaline treatment.

\section{INTRODUCCIÓN}

El caolín es un material arcilloso constituido mayoritariamente por caolinita, la cual es un aluminosilicato laminar del tipo 1:1 $\left(\mathrm{Al}_{2} \mathrm{Si}_{2} \mathrm{O}_{5}(\mathrm{OH})_{4}\right)$, donde una capa tetraédrica de silica se encuentra unida a una capa octaédrica de alúmina formando láminas que se mantienen unidas por enlaces de hidrógeno. Las aplicaciones del caolín abarcan un amplio abanico, desde la industria de la cerámica hasta la catálisis heterogénea $(1,2)$. Éstas se encuentran controladas por diversos factores incluyendo el origen geológico del material de partida, su composición mineralógica y sus propiedades químicas y ópticas (color, brillo, tamaño de partícula, etc.) (3).

La importancia industrial del caolín suele conllevar la necesidad de realizar una previa purificación o mejora del mismo, tratando, así, de aumentar su utilidad. Entre las técnicas más comunes destacan: 1) flotación, para la mejora del brillo; 2) separación magnética, para la eliminación de metales; 3 ) ozonización, para la eliminación del material orgánico; 4) molienda, afecta a la estructura y al tamaño de partícula; 5) calcinación, permite modificar la estructura y obtener sólidos con mejor hidrofobicidad y buenas propiedades ópticas y eléctricas (4).

Trabajos previos han mostrado la posibilidad de modificar las arcillas laminares mediante tratamientos químicos, como cambio iónico, reacción con ácidos o pilarización, y tratamientos físicos, como liofilización o ultrasonidos, consiguiéndose materiales de elevado valor añadido (5). Sin embargo, sólo algunos tratamientos son capaces de modificar al caolín debido a la inercia química que su estructura pro- voca. Por ello, se han estudiado mecanismos que consiguen modificar previamente al caolín, sin elevar excesivamente el coste del producto, obteniéndose sólidos más susceptibles de ser modificados. Entre estos mecanismos cabe destacar la molienda $(6,7)$ y la calcinación (8). En este último caso, las propiedades de los productos dependen de la temperatura de calcinación empleada, destacando: 1) $550^{\circ} \mathrm{C}$ - metacaolín $\left.\left(\mathrm{Al}_{2} \mathrm{Si}_{2} \mathrm{O}_{7}\right) ; 2\right) 925^{\circ} \mathrm{C}-950^{\circ} \mathrm{C}$ - espinela $\left(\mathrm{Si}_{3} \mathrm{Al}_{4} \mathrm{O}_{12}\right)$; 3$) 1000-1100^{\circ} \mathrm{C}$ - mullita $\left(\mathrm{Si}_{2} \mathrm{Al}_{6} \mathrm{O}_{13}\right)$; los cuales presentan, por sí mismos, importantes aplicaciones (papel, PVC, plásticos, etc.) (9).

Debido a la importancia industrial del caolín y con el objetivo de obtener materiales con propiedades tecnológicas a partir del mismo, este trabajo está dedicado al estudio del efecto que la activación ácida y básica producen sobre este material. La activación ácida es un método empleado para mejorar las propiedades superficiales de las arcillas. Consiste en tratar la arcilla con disoluciones de ácidos inorgánicos capaces de modificar su composición y su estructura, cuyo resultado depende de las variables del tratamiento y del material de partida (10). En cuanto a la activación básica, puede ser definida como el tratamiento del material arcilloso con disoluciones alcalinas. Cabe destacar que, a pesar de ser un método poco empleado sobre arcillas, se han encontrado trabajos que describen la síntesis de zeolitas mediante tratamientos del caolín con disoluciones alcalinas de hidróxidos metálicos a una temperatura conveniente $(11,12)$. En el presente trabajo se llevará a cabo un estudio detallado sobre la síntesis 
de metacaolín por calcinación del caolín y sobre la posterior activación ácida y básica de dichos sólidos, haciendo hincapié en las condiciones de los tratamientos y realizando una caracterización completa de los sólidos obtenidos.

\section{EXPERIMENTAL}

\subsection{Preparación de las muestras}

El caolín natural utilizado en este trabajo procede del yacimiento de Navalacruz, Zamora. Su estudio inicial reveló la presencia de cuarzo, mica y esmectita como impurezas mineralógicas, las cuales fueron casi totalmente eliminadas mediante la extracción de la fracción inferior a dos micras. Este caolín purificado fue calcinado a 600, 700,800 y $900^{\circ} \mathrm{C}$, con el fin de obtener los metacaolines. La duración del tratamiento fue de $10 \mathrm{~h}$, realizado en atmósfera ambiente con una velocidad de calentamiento de $10^{\circ} \mathrm{C} / \mathrm{min}$. La nomenclatura empleada para estos metacaolines fue MK-T, siendo $T$ la temperatura de calcinación empleada.

La activación ácida de los metacaolines se realizó con disoluciones de $\mathrm{HCl} 6 \mathrm{M}$, a $90^{\circ} \mathrm{C}$, en condiciones de reflujo, durante $6,12,18$ y $24 \mathrm{~h}$. Para ello, se tomaron $6 \mathrm{~g}$ del sólido poniéndose en contacto con $180 \mathrm{~mL}$ de la disolución ácida, durante los tiempos indicados y bajo agitación constante. Los sólidos obtenidos fueron separados por centrifugación, lavados hasta la eliminación de los aniones cloruro y secados en estufa a $70^{\circ} \mathrm{C}$ durante una noche. La nomenclatura empleada para estas muestras fue MK-T-HCl-t, donde $t$ indica el tiempo del tratamiento ácido.

Para la activación básica, se emplearon disoluciones de $\mathrm{KOH} 5 \mathrm{M}$, realizando el tratamiento a $90^{\circ} \mathrm{C}$, en condiciones de reflujo, durante 6 y 24 h mantenido una agitación constante. La relación sólido/disolución fue la misma que la empleada en la activación ácida, $30 \mathrm{ml} / \mathrm{g}$. Los sólidos obtenidos se separaron y lavaron por centrifugación, hasta la neutralización de las aguas de lavado, y posteriormente se secaron en estufa a $70^{\circ} \mathrm{C}$ durante una noche. La denominación empleada para estos sólidos fue MK-T-KOH-t, siendo $t$ el tiempo de duración del tratamiento básico.

\subsection{Técnicas de Caracterización}

La composición química fue determinada por Espectrometría de Emisión de Plasma Inductivamente Acoplada (ICPS), en los laboratorios “Activation Laboratories Ltd." (Ancaster, Ontario, Canadá).

Los difractogramas de rayos $\mathrm{X}$ fueron realizados en un difractómetro Siemens D500 conectado a un microprocesador DACO-MP, a $40 \mathrm{kV}$ y $30 \mathrm{~mA}$, empleando monocromador de grafito para la radiación $\mathrm{CuK} \alpha$. La identificación estructural de los sólidos fue realizada empleándose los ficheros JCPDS.

El análisis térmico diferencial (ATD) y el análisis termogravimétrico (TG) se midieron en un equipo Perkin-Elmer DTA7 y en una termobalanza Perkin-Elmer TGA7, respectivamente. Ambos se realizaron en atmósfera de $\mathrm{O}_{2}$ incrementando la temperatura hasta $1000^{\circ} \mathrm{C}$ con una velocidad de calentamiento de $10^{\circ} \mathrm{C} / \mathrm{min}$.

Los espectros de infrarrojo fueron registrados en un espectrómetro FT-IR Perkin-Elmer 1730 sobre pastillas de $\mathrm{KBr}$, diluyendo la muestra en bromuro potásico en una proporción de 1/300.

Las isotermas de adsorción-desorción de nitrógeno a 77K se midieron en un equipo volumétrico automático ASAP 2000. Las muestras fueron pretratadas mediante desgasificación, primero a temperatura ambiente durante $1 \mathrm{~h}$ y posteriormente a $110^{\circ} \mathrm{C}$ durante $2 \mathrm{~h}$. Las con- diciones del análisis empleadas fueron: 1) $\mathrm{P} / \mathrm{P}_{0}<0,1$ inyección de dosis controladas de $4 \mathrm{~cm}^{3}$ de $\mathrm{N}_{2} / \mathrm{g}$ (CNPT) programando un tiempo de estabilización de 1-1,5 h; 2) $\mathrm{P} / \mathrm{P}_{0}>0,1$ utilización de una tabla de presiones relativas prefijadas entre 0,12 y 0,99 , a intervalos de $0,02-0,05$. El área superficial fue calculada por el método BET (13), el diámetro de poro medio mediante el método Dubinin (14) y la representación " $\mathrm{t}$ " por el método de De Boer (15).

El estudio por microscopía electrónica de barrido se llevó a cabo en un microscopio Digital Scanning Microscope Zeiss DSM 940. Para ello las muestras fueron recubiertas previamente con una capa de oro para hacerlas conductoras, empleando el sistema Bio-Rad ES100 SEN Coating System.

\section{RESULTADOS Y DISCUSIÓN}

\subsection{Síntesis de metacaolines}

La calcinación del caolín entre 600 y $900^{\circ} \mathrm{C}$ da lugar a la formación de sólidos amorfos. Sus difractogramas de rayos $\mathrm{X}$ muestran la desaparición de los picos propios de la caolinita, presentando un halo entre $15-30^{\circ}$ de $2 \theta$ atribuido a la presencia de sílice amorfa. Por su parte, la espectroscopía infrarroja mostró la pérdida de los grupos hidroxilos de la caolinita (tensión Al-OH: $3700-3600 \mathrm{~cm}^{-1}$; deformación Al-OH-Al: 900-930 $\mathrm{cm}^{-1}(16)$ ), así como la modificación de las bandas atribuidas a los enlaces Si-O (tensión: 1100-1000 $\mathrm{cm}^{-1}$ ) (17). Estas modificaciones son causadas por la deshidroxilación producida sobre el caolín por el tratamiento térmico, la cual va acompañada de la modificación de los entornos del $\mathrm{Al}$ y del $\mathrm{Si}$, como ha sido explicado por diversos autores mediante RMN observando el cambio del aluminio hexa-coordinado a penta- y tetra-coordinado $(18,19)$. Estos efectos causan la deformación de las láminas del caolín y su consiguiente pérdida de cristalinidad.

La superficie específica de los metacaolines $\left(\approx 10 \mathrm{~m}^{2} / \mathrm{g}\right)$ es inferior a la del caolín de partida $\left(18 \mathrm{~m}^{2} / \mathrm{g}\right)$, debido a la distorsión sufrida durante la calcinación que puede producir la sinterización de las partículas. Las cuatro temperaturas empleadas dan lugar a sólidos con características estructurales similares, tan sólo el MK-900 posee una superficie específica ligeramente inferior probablemente debido a la sinterización de las partículas que la elevada temperatura puede causar.

\subsection{Activación ácida}

La composición química de los sólidos tratados con la disolución ácida a $90^{\circ} \mathrm{C}$ se presenta en la tabla 1 , referida a las muestras secas y comparada con la del caolín de partida. Se observa como el tratamiento ácido produce la disolución de los cationes $\mathrm{Al}^{3+}$ de la capa octaédrica del caolín, lo cual conlleva un aumento del contenido en $\mathrm{SiO}_{2}$. El ataque del ácido sobre el metacaolín resulta ser más efectivo cuanto mayor es la duración del tratamiento, a $6 \mathrm{~h}$ se consigue disolver aproximadamente el 90\% del $\mathrm{Al}^{3+}$ para los metacaolines MK-600, MK700 y MK-800, alcanzándose el 95\% al aumentar el tiempo de contacto a $24 \mathrm{~h}$ donde el contenido de $\mathrm{Al}_{2} \mathrm{O}_{3}$ tan sólo es del $2 \%$, aproximadamente. El MK-900 resulta ser el metacaolín menos afectado por la activación ácida, tras el tratamiento durante $6 \mathrm{~h}$ el sólido mantiene el 30\% de $\mathrm{Al}_{2} \mathrm{O}_{3^{\prime}}$ porcentaje que disminuye muy poco cuando la duración del tratamiento aumenta hasta $24 \mathrm{~h}$. Esto supone que la calcinación entre $600-800^{\circ} \mathrm{C}$ da lugar a sólidos más reactivos, mientras que el empleo de mayores temperaturas perjudica dicha reactividad. Probablemente durante la calcinación a $900^{\circ} \mathrm{C}$ tiene lugar cierta sinterización de las partículas del caolín, convirtiéndolas en más inaccesibles ante los protones del ácido. 
TABLA 1. COMPOSICIÓN QUÍMICA, REFERIDA A LAS MUESTRAS SECAS, DEL CAOLÍN DE PARTIDA, DE LOS SÓLIDOS OBTENIDOS TRAS LOS TRATAMIENTOS ÁCIDOS Y DE LOS SÓLIDOS SOMETIDOS A TRATAMIENTO BÁSICO.

\begin{tabular}{|l|c|c|c|c|c|c|c|c|}
\hline Muestra & $\mathrm{SiO}_{2}$ & $\mathrm{Al}_{2} \mathrm{O}_{3}$ & $\mathrm{Fe}_{2} \mathrm{O}_{3}$ & $\mathrm{MgO}$ & $\mathrm{K}_{2} \mathrm{O}$ & $\mathrm{TiO}_{2}$ & $\mathrm{CaO}$ & $\mathrm{Na}_{2} \mathrm{O}$ \\
\hline Caolín & 55,72 & 39,83 & 2,47 & 0,52 & 0,76 & 0,37 & 0,24 & 0,09 \\
\hline MK-600-HCl-6 & 89,08 & 7,86 & 0,35 & 0,34 & 1,37 & 0,87 & 0,07 & 0,06 \\
\hline MK-700-HCl-6 & 92,36 & 5,35 & 0,32 & 0,20 & 0,80 & 0,89 & 0,04 & 0,04 \\
\hline MK-800-HCl-6 & 90,71 & 6,80 & 0,51 & 0,15 & 0,83 & 0,88 & 0,06 & 0,05 \\
\hline MK-900-HCl-6 & 65,62 & 30,34 & 1,79 & 0,35 & 1,02 & 0,66 & 0,15 & 0,06 \\
\hline MK-600-HCl-12 & 94,21 & 3,34 & 0,22 & 0,22 & 0,89 & 0,86 & 0,17 & 0,09 \\
\hline MK-600-HCl-18 & 95,09 & 2,81 & 0,16 & 0,16 & 0,79 & 0,83 & 0,10 & 0,07 \\
\hline MK-600-HCl-24 & 94,81 & 3,20 & 0,12 & 0,12 & 0,85 & 0,83 & 0,02 & 0,05 \\
\hline MK-700-KCl-24 & 96,81 & 1,76 & 0,11 & 0,09 & 0,35 & 0,84 & 0,01 & 0,02 \\
\hline MK-800-HCl-24 & 97,21 & 1,44 & 0,13 & 0,06 & 0,27 & 0,87 & 0,01 & 0,01 \\
\hline MK-900-HCl-24 & 72,09 & 24,75 & 1,47 & 0,32 & 0,55 & 0,65 & 0,13 & 0,04 \\
\hline MK-600-KOH-6 & 37,30 & 26,75 & 1,47 & 0,32 & 33,39 & 0,41 & 0,16 & 0,21 \\
\hline MK-700-KOH-6 & 35,17 & 25,51 & 1,42 & 0,31 & 36,82 & 0,40 & 0,16 & 0,21 \\
\hline MK-800-KOH-6 & 35,57 & 26,26 & 1,42 & 0,32 & 35,64 & 0,41 & 0,16 & 0,21 \\
\hline MK-900-KOH-6 & 33,22 & 33,30 & 1,81 & 0,38 & 30,41 & 0,49 & 0,20 & 0,19 \\
\hline MK-600-KOH-24 & 39,34 & 28,90 & 1,53 & 0,33 & 29,15 & 0,45 & 0,16 & 0,15 \\
\hline MK-700-KOH-24 & 36,91 & 27,90 & 1,46 & 0,31 & 32,70 & 0,40 & 0,17 & 0,15 \\
\hline MK-800-KOH-24 & 32,26 & 24,23 & 1,24 & 0,27 & 41,27 & 0,37 & 0,13 & 0,21 \\
\hline MK-900-KOH-24 & 33,09 & 25,98 & 1,38 & 0,29 & 38,48 & 0,44 & 0,15 & 0,19 \\
\hline
\end{tabular}

Los difractogramas de rayos $\mathrm{X}$ de estos sólidos, representados en la figura 1, presentan el mismo halo a $2 \theta$ entre 15 y $30^{\circ}$ que sus metacaolines de partida, indicando la presencia de sílice amorfa. Este halo posee mayor intensidad a medida que aumenta la duración del tratamiento, coincidiendo con los valores de composición química descritos anteriormente, la capa alumínica se disuelve más aumentando, así, el contenido en sílice. Junto a este halo amorfo, se observa la presencia de los picos atribuidos a las impurezas mineralógicas, cuarzo y mica, las cuales no fueron eliminadas totalmente durante la purificación, indicando la estabilidad de dichas especies ante el tratamiento ácido agresivo.

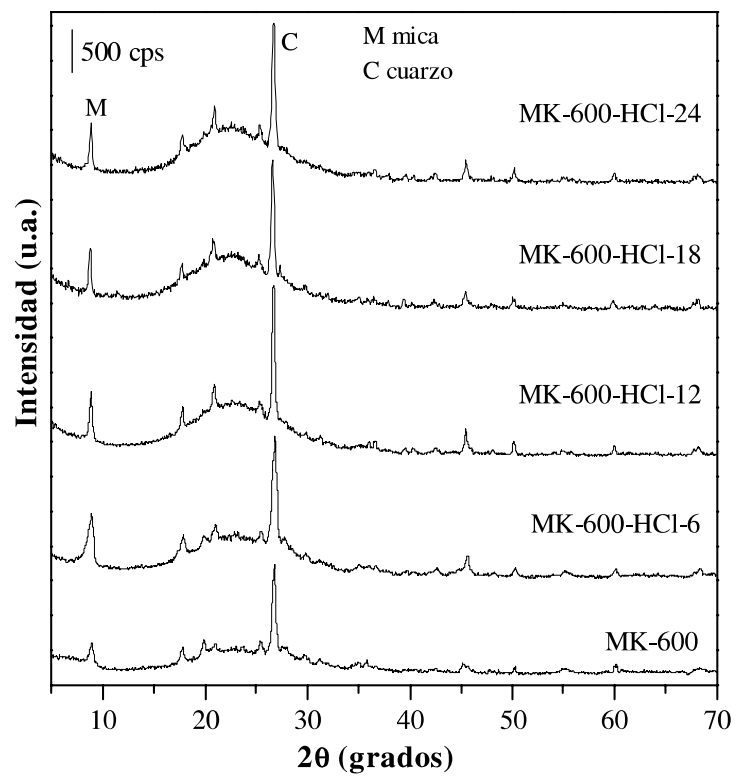

Figura 1. Difractogramas de rayos X del MK-600 y de los sólidos obtenidos tras su activación ácida MK-600-HCl-t.

La modificación sufrida por los metacaolines durante el tratamiento ácido también puede observarse gracias a los análisis térmicos. Los metacaolines de partida no presentan efectos térmicos relevantes, incluso su análisis termogravimétrico refleja un contenido en agua escaso. Sin embargo, los sólidos activados muestran unas curvas termo- gravimétricas divididas en dos etapas (figura 2.A): la primera centrada a $100^{\circ} \mathrm{C}$, supone pérdidas de peso entre $4-8 \%$ en función de la duración del tratamiento; mientras la segunda se produce de forma gradual, entre $250-900^{\circ} \mathrm{C}$, perdiendo entre $3-5 \%$ de peso. El primer efecto es debido a la pérdida del agua adsorbida en los sólidos, mientras que el segundo se atribuye a la pérdida de los hidroxilos de las especies silanoles, $\mathrm{Si}(\mathrm{OSi})_{3} \mathrm{OH}$, formadas durante el tratamiento ácido (20).
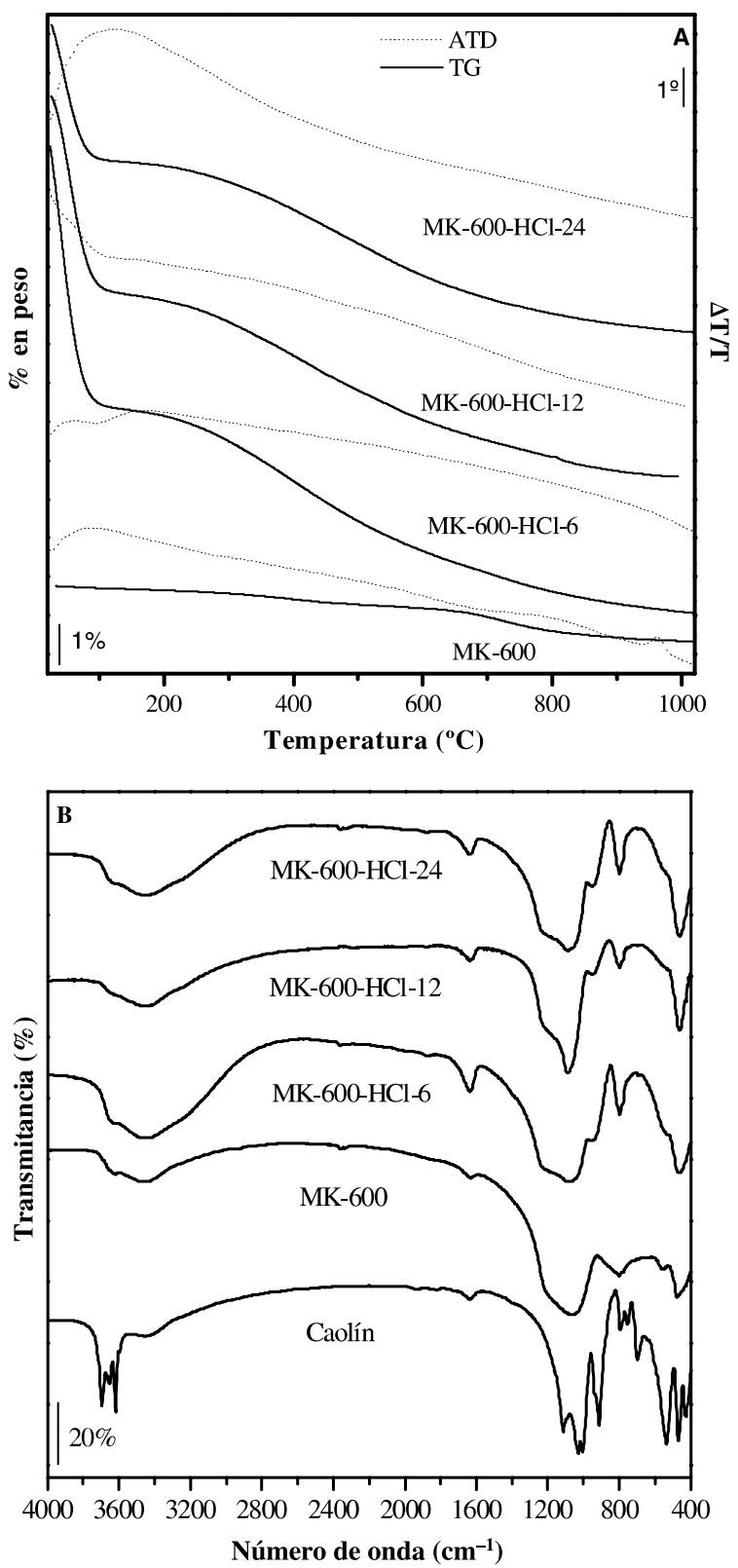

Figura 2. A) Curvas térmicas del MK-600 y MK-600-HCl-t siendo $t, 6$ 12 y 24 h. B) Espectros de infrarrojo del caolín, MK-600 y MK-600-HCl$t$ siendo $t, 6,12$ y $24 \mathrm{~h}$.

Estos efectos también son evidentes al estudiar los espectros de infrarrojo de estos sólidos activados, presentados en la figura 2.B. Por un lado, se comprueba como el contenido en agua de los sólidos tratados es mayor que el de los metacaolines de partida, ya que la intensidad de sus bandas ( 3460 y $1630 \mathrm{~cm}^{-1}$ ) aumenta; por otro lado, aparece una nueva banda a $950 \mathrm{~cm}^{-1}$ atribuida a la vibración de tensión del enlace $\mathrm{Si}-\mathrm{OH}$ de las especies $\left(\mathrm{Si}_{3}\right) \mathrm{Si}-\mathrm{OH}(21)$, cuya ausencia en los metacaolines originales corrobora la formación de nuevas especies durante el tratamiento ácido. La formación de estas especies está controlada por la duración 
del tratamiento ácido, ya que la intensidad de su banda aumenta a medida que aumentamos el tiempo de la activación. Los análisis térmicos y la espectroscopía infrarroja vuelven a mostrar la menor reactividad del MK-900 ante la activación ácida, puesto que tanto sus curvas térmicas como su espectro de infrarrojo son análogos a los obtenidos para los metacaolines de partida (ausencia de etapas y baja pérdida de peso en el TG; ausencia de la banda del Si-OH en el espectro de IR).

Las propiedades texturales de los sólidos activados son recogidas en la tabla 2, calculadas a partir de las isotermas de adsorción-desorción de nitrógeno a 77K y de las representaciones " $\mathrm{t}$ ". Se puede observar como el tratamiento ácido realizado durante $6 \mathrm{~h}$ produce un considerable aumento de la superficie específica de los metacaolines MK-600, MK-700 y MK-800, aumentando de $10 \mathrm{~m}^{2} / \mathrm{g}$ a valores de $200 \mathrm{~m}^{2} / \mathrm{g}$. El tipo de isoterma y los datos obtenidos a partir de la representación " $\mathrm{t}$ " reflejan como dicho aumento es debido al desarrollo de la superficie externa e interna de los sólidos, pudiéndose decir que durante el tratamiento ácido se produce la deslaminización del metacaolín junto a la formación de sílice porosa. La microporosidad desarrollada en estos sólidos es debida al reordenamiento sufrido por las capas silícicas tras la disolución de las capas alumínicas. Los tetraedros de $\mathrm{SiO}_{4}$ sufren rotaciones dentro de sus propias láminas dando lugar a nuevas ordenaciones estructurales que permiten la existencia de poros en su interior, de aproximadamente $16 \AA$ (20). Por su parte, los resultados reflejan la menor reactividad del MK-900 ante el tratamiento ácido. La superficie específica del sólido tratado es inferior a la de los demás sólidos, aunque superior a la del metacaolín de partida, no llegando a desarrollar microporosidad.

Tabla 2. Propiedades texturales del MK-600 y de los sólidos obteNIDOS POR ACTIVACIÓN ÁCIDA Y BÁSICA $\left(\mathrm{S}_{\text {BET' }^{\prime}}\right.$ SUPERFICIE ESPECÍFICA; $\mathrm{S}_{\text {EXY' }}$ SUPERFICIE EXTERNA; $S_{M P}$ SUPERFICIE DE MICROPOROS; $V_{M P}$ VOLUMEN DE MICROPOROS Y $\mathrm{D}_{\mathrm{r}^{\prime}}$ DIÁMETRO DE PORO MEDIO).

\begin{tabular}{|l|c|c|c|c|c|}
\hline Muestra & $\mathrm{S}_{\mathrm{BET}}\left(\mathrm{m}^{2} / \mathrm{g}\right)$ & $\mathrm{S}_{\mathrm{EXT}}\left(\mathrm{m}^{2} / \mathrm{g}\right)$ & $\mathrm{S}_{\mathrm{MP}}\left(\mathrm{m}^{2} / \mathrm{g}\right)$ & $\mathrm{V}_{\mathrm{MP}}\left(\mathrm{cm}^{3} / \mathrm{g}\right)$ & $\mathrm{D}_{\mathrm{P}}(\AA)$ \\
\hline MK-600 & 10,6 & 9,8 & 0,8 & 0,0003 & \\
\hline MK-600-HCl-6 & 219 & 85 & 135 & 0,0643 & 15,08 \\
\hline MK-700-HCl-6 & 172 & 126 & 46 & 0,0223 & 19,05 \\
\hline MK-800-HCl-6 & 209 & 104 & 105 & 0,0511 & 15,96 \\
\hline MK-900-HCl-6 & 59 & 59 & 0,4 & 0,0001 & --- \\
\hline MK-600-HCl-12 & 25 & 23 & 2,5 & 0,0011 & \\
\hline MK-600-HCl-18 & 24 & 21 & 2,7 & 0,0013 & \\
\hline MK-600-HCl-24 & 22 & 20 & 1,6 & 0,0007 & \\
\hline MK-700-KCl-24 & 22 & 18,4 & 3,5 & 0,0017 & \\
\hline MK-800-HCl-24 & 23 & 20 & 2,9 & 0,0014 & \\
\hline MK-900-HCl-24 & 23 & 21 & 1,8 & 0,0008 & \\
\hline MK-600-KOH-6 & 4,1 & 4,1 & --- & --- & \\
\hline MK-700-KOH-6 & 3,7 & 3,7 & --- & --- & \\
\hline MK-800-KOH-6 & 3,6 & 2,4 & 1,2 & 0,0006 & \\
\hline MK-900-KOH-6 & 16,5 & 16,5 & --- & --- & \\
\hline MK-600-KOH-24 & 2,4 & & & & \\
\hline MK-700-KOH-24 & 3,4 & & & & \\
\hline MK-800-KOH-24 & 3,7 & & & & \\
\hline MK-900-KOH-24 & 0,1 & & & & \\
\hline \multicolumn{7}{|l|}{} & & & & \\
\hline
\end{tabular}

El empleo de tiempos de activación superiores a $6 \mathrm{~h}$ supone la disminución del área superficial de los sólidos activados, junto a la desaparición de la microporosidad que había sido formada al principio del tratamiento. Este efecto es observable tanto a 12 como a $24 \mathrm{~h}$ de tratamiento, aunque los sólidos presentan valores de $S_{\mathrm{BET}}$ superiores a sus metacaolines de partida resultan ser considerablemente inferiores a los valores de los sólidos tratados durante 6 h. Esta disminución puede ser ocasionada por el colapso de los microporos formados al principio del tratamiento, ya que la mayor disolución del $\mathrm{Al}_{2} \mathrm{O}_{3}$ ocasiona una mayor modificación de las capas silícicas, pudiendo producirse reacciones de condensación entre las especies que forman las partículas de $\mathrm{SiO}_{2}$, variando, así, su morfología final.
Las microfotografías obtenidas por microscopía electrónica de barrido, presentadas en la figura 3, reflejan la formación de partículas amorfas a partir de las láminas del metacaolín durante el tratamiento ácido. Dichas partículas coexisten con láminas originales que parecen no haber sido modificadas, a pesar de que su composición química mostraba un elevado grado de disolución del $\mathrm{Al}_{2} \mathrm{O}_{3}$. El parecido encontrado entre las microfotografías de los sólidos tratados durante 6 y $24 \mathrm{~h}$ no muestran las diferencias de composición, estructura y superficie que se evidencian por otras técnicas.
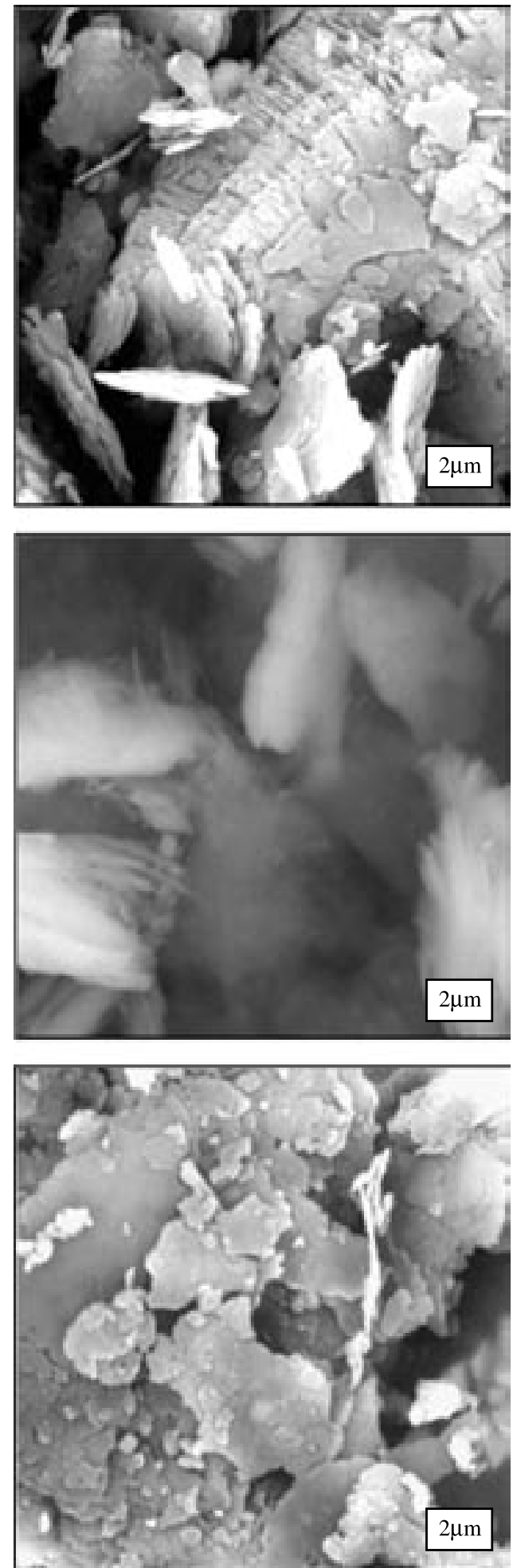

Figura 3. Microfotografías de MEB del MK-600 (superior), MK-600$\mathrm{HCl}-6$ (centro) y MK-600-HCl-24 (inferior). 


\subsection{Activación básica}

Los primeros estudios sobre el comportamiento de los metacaolines ante disoluciones alcalinas fueron realizados empleando disoluciones más diluidas y a menor temperatura. Estas condiciones resultaron ser poco eficaces para modificar a los metacaolines, escogiéndose, por ello, las condiciones mencionadas anteriormente como las más óptimas para estos estudios, $\mathrm{KOH} 5 \mathrm{M}$ a $90^{\circ} \mathrm{C}$. Bajo tales condiciones los metacaolines sufrieron una importante modificación, los difractogramas de rayos $\mathrm{X}$ presentados en la figura 4.A reflejan el cambio estructural producido durante el tratamiento alcalino. Se observa como el difractograma del metacaolín, propio de un sólido amorfo, se convierte en un difractograma compuesto por un amplio número de picos, los cuales fueron identificados como los característicos de la zeolita K-F (Z) (22). Estos difractogramas muestran también como la síntesis de la zeolita se produce a partir de los cuatro metacaolines, no parece existir una temperatura de calcinación del caolín preferente para su formación. Algo similar ocurre con el tiempo de duración del tratamiento, los sólidos obtenidos presentan la misma estructura tanto si han sido obtenidos por activación durante 6 o 24 h.
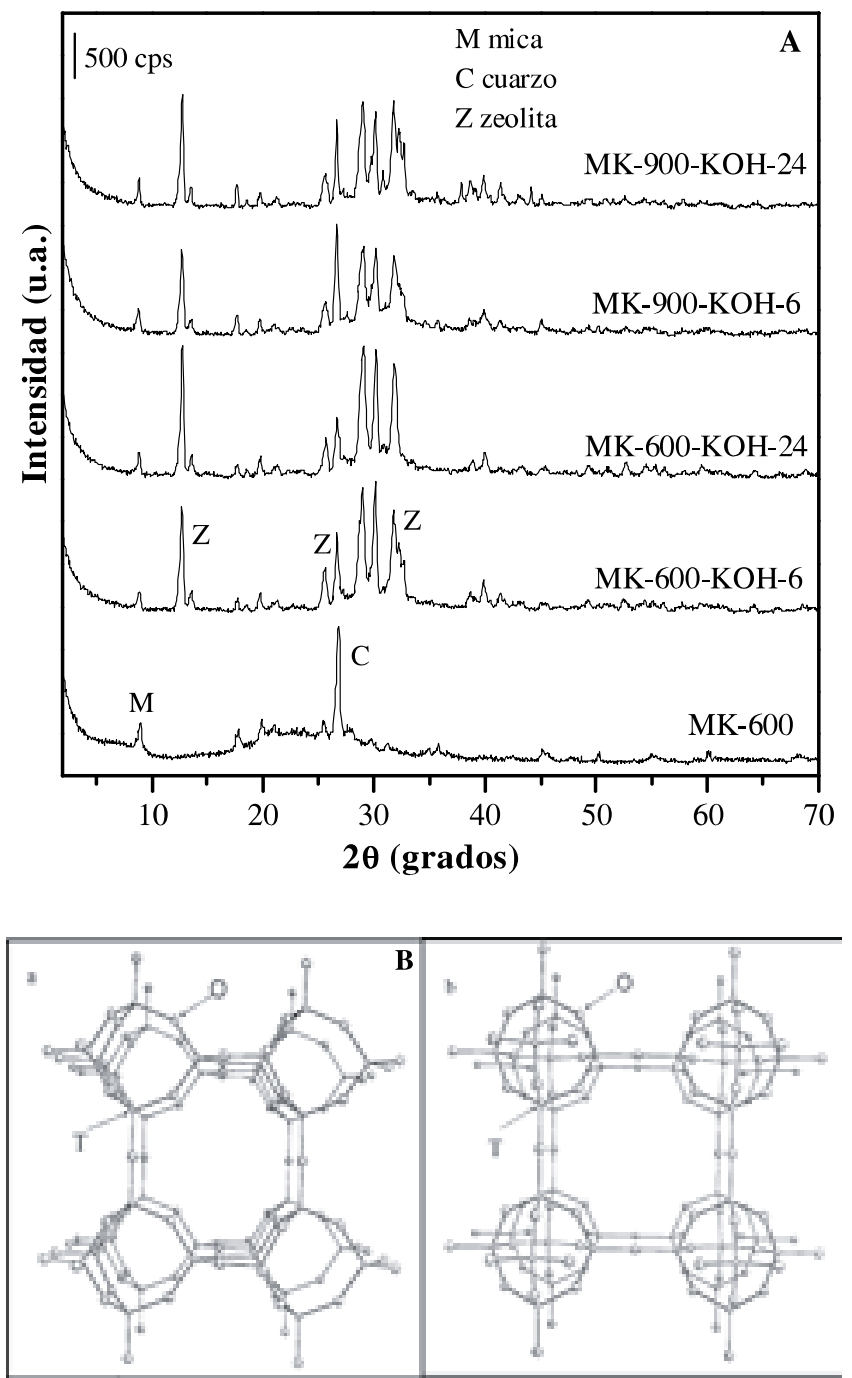

Figura 4. A) Difractogramas de rayos X del MK-600 y de los sólidos obtenidos tras su activación básica MK-T-KOH- $t(T=600,900)$; B) Representación de la estructura tipo de la edingtonita, a) vista a lo largo de la dirección [100] y b) desde la dirección [001].
La cristalización de zeolitas por tratamiento alcalino de metacaolín ha sido descrita en la literatura, utilizándose temperaturas cercanas a los $100^{\circ} \mathrm{C}$ para su síntesis e incluso, en ocasiones, usándose autoclaves para llevar a cabo el tratamiento hidrotermal (22). El tipo de zeolita formada depende de la composición de la mezcla de reacción, por ejemplo depende de la naturaleza del caolín de partida, del tipo de base empleada $\left(\mathrm{NaOH}, \mathrm{KOH}\right.$ o $\left.\mathrm{Ba}(\mathrm{OH})_{2}\right)$, e incluso de si se añade sílice o algún silicato para variar la relación Si/Al (10). En nuestro trabajo tan sólo obtuvimos la zeolita K-F, ésta posee una estructura análoga a la que posee la edingtonita. En ella, los tetraedros $\mathrm{TO}_{4}(\mathrm{~T}=\mathrm{Si}, \mathrm{Al})$ se enlazan entre sí dando lugar a la estructura presentada en la figura 4.B, esta disposición crea aberturas o anillos de ocho lados que dan lugar a la formación de los canales característicos de los materiales zeolíticos (23).

La composición química de estos sólidos, recogida en la tabla 1, muestra la disminución tanto del contenido en $\mathrm{SiO}_{2}$ como del $\mathrm{Al}_{2} \mathrm{O}_{3}$ en torno a un $30 \%$, acompañado de un aumento similar en el contenido de $\mathrm{K}_{2} \mathrm{O}$. Estas variaciones son debidas a la formación de la zeolita K-F, identificada por rayos X, cuya composición química teórica es: 37,99\% $\mathrm{SiO}_{2}, 32,23 \% \mathrm{Al}_{2} \mathrm{O}_{3}$ y 29,28\% $\mathrm{K}_{2} \mathrm{O}$. Al comparar los datos experimentales con el teórico se observa como el contenido de $\mathrm{SiO}_{2}$ es bastante similar, mientras que el porcentaje de $\mathrm{Al}_{2} \mathrm{O}_{3}$ es ligeramente inferior y el de $\mathrm{K}_{2} \mathrm{O}$ más elevado del teórico. El menor contenido en $\mathrm{Al}_{2} \mathrm{O}_{3}$ puede deberse a la sustitución isomórfica del $\mathrm{Al}^{3+}$ por $\mathrm{Fe}^{3+}$ en la estructura de la zeolita, ya que el porcentaje de $\mathrm{Fe}_{2} \mathrm{O}_{3}$ en la composición es considerable. En cuanto al potasio, éste se encuentra como catión de cambio, existiendo un catión por cada sustitución isomórfica del $\mathrm{Si}^{4+}$ por $\mathrm{Al}^{3+}$ $\left(\mathrm{Fe}^{3+}\right)$, de manera que el resto del potasio puede encontrarse fijado al sólido como $\mathrm{K}_{2} \mathrm{O}$ o $\mathrm{K}_{2} \mathrm{CO}_{3}$.

Esta última afirmación se basa en el estudio de los espectros de infrarrojo de los metacaolines activados, los cuales se muestran en la figura 5. En ellos es posible observar la presencia de una nueva banda a $1440 \mathrm{~cm}^{-1}$ asignada a la tensión asimétrica del $\mathrm{CO}_{3}{ }^{2-}(17)$, la cual refleja la formación de $\mathrm{K}_{2} \mathrm{CO}_{3}$ por reacción del $\mathrm{CO}_{2}$ atmosférico con la disolución fuertemente alcalina empleada en la activación, ya que no se tomaron precauciones especiales para el aislamiento del medio de reacción de la atmósfera.

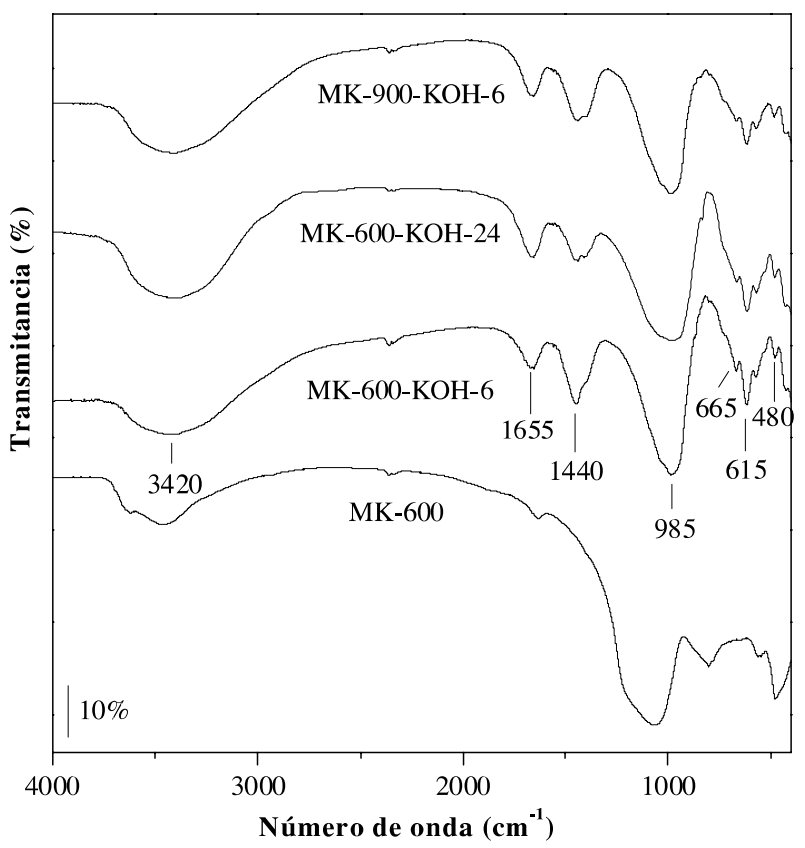

Figura 5. Espectros de infrarrojo del MK-600, MK-600-KOH-6, MK600-KOH-24 y MK-900-KOH-24. 
Estos espectros reflejan de nuevo la modificación sufrida por los metacaolines tras el tratamiento alcalino, permitiendo completar la caracterización de los sólidos sintetizados. Se observa como la banda a $1090 \mathrm{~cm}^{-1}$ atribuida a la tensión del Si-O del metacaolín se desplaza a menores números de onda, $985 \mathrm{~cm}^{-1}$. Esta banda y las que aparecen a inferiores números de onda son atribuidas a la formación de la estructura tipo zeolita. Los espectros de infrarrojo de este tipo de materiales están constituidos por unas bandas debidas a las vibraciones internas de los $\mathrm{TO}_{4}$ y por otras atribuidas a las vibraciones relacionadas con las uniones entre los tetraedros que dan lugar a la estructura final de la zeolita. Así, a 985, 665 y $480 \mathrm{~cm}^{-1}$ aparecen las vibraciones de los enlaces $\mathrm{T}-\mathrm{O}(\mathrm{T}=\mathrm{Si}, \mathrm{Al})$ de los tetraedros internos, correspondiendo a la tensión asimétrica, tensión simétrica y deformación, respectivamente; a su vez, el resto de las bandas mencionadas se deben al movimiento de los anillos, poros y aperturas que la disposición de las unidades tetraédricas genera (24). Junto a las bandas descritas se observan aquellas atribuidas al agua adsorbida $\left(3420,1655 \mathrm{~cm}^{-1}\right)$, las cuales presentan mayor intensidad que las del metacaolín de partida.

El mayor contenido en agua de estos sólidos también se evidenció en sus análisis térmicos, en ellos se produce un efecto endotérmico que abarca desde la temperatura ambiente hasta $400^{\circ} \mathrm{C}$, aunque se encuentra centrado a $105^{\circ} \mathrm{C}$, al que le corresponde una pérdida de peso del $9 \%$, aproximadamente. Este proceso se atribuye a la pérdida del agua presente en la estructura de la zeolita, la cual puede encontrarse en el interior de sus canales y como agua de constitución de la misma. La modificación de los metacaolines durante estos tratamientos no sólo se muestra en su mayor contenido en agua sino en la desaparición del cambio de fase propio del metacaolín, que ocurría a $960^{\circ} \mathrm{C}$ al transformarse en fase tipo espinela, puesto que la zeolita K-F se descompone a temperaturas superiores, $1095^{\circ} \mathrm{C}$, dando lugar a la formación de kaliofilita (25).

La caracterización de los metacaolines sometidos al tratamiento alcalino muestra el gran parecido entre todos los sólidos sintetizados, la composición química es similar, así como sus espectros de infrarrojo. La variación de la temperatura de calcinación a la que el caolín original fue calcinado no influye de forma apreciable en el tratamiento alcalino, como tampoco afecta de forma excesiva el aumento de la duración del mismo. Este comportamiento se debe a la forma en la que la disolución alcalina ataca a los metacaolines. Al principio de la reacción la disolución alcalina modifica a los metacaolines disolviendo el $\mathrm{SiO}_{2}$ y el $\mathrm{Al}_{2} \mathrm{O}_{3}$ de su estructura. Estas especies permanecen en la disolución formando un gel amorfo a medida que su concentración aumenta, lo cual depende de la concentración del hidróxido alcalino empleado. Si el medio es suficientemente básico, las concentraciones de estas especies en disolución pueden alcanzar los valores adecuados para que el gel amorfo cristalice, con incorporación del catión alcalino en la estructura, formándose así un aluminosilicato cristalino, cuya estructura varía en función de la composición del medio de reacción (11). En este estudio, al emplear disoluciones de $\mathrm{KOH} 5 \mathrm{M}$ los cuatro metacaolines de partida fueron disueltos de forma similar, cristalizando junto con el $\mathrm{K}^{+}$de la disolución en la misma estructura tras $6 \mathrm{~h}$ de tratamiento, manteniéndose al aumentar la duración a $24 \mathrm{~h}$.

Las propiedades texturales de los metacaolines tratados con disoluciones alcalinas son mostradas en la tabla 2, se observa como estos sólidos presentan áreas superficiales inferiores a las de sus metacaolines de partida con ausencia de microporosidad. Aunque los materiales zeolíticos estén constituidos por canales y cavidades en su interior que pueden generar microporosidad, en ocasiones éstos pueden presentar una baja accesibilidad hacia las moléculas de adsorbato por el tamaño de sus partículas, con lo que la difusión del adsorbato durante la adsorción se ralentiza e impide la identificación de microporos (26). Ésta sería la causa por la que los datos obtenidos a partir de las repre- sentaciones " $\mathrm{t}$ ” y de las isotermas de adsorción-desorción de nitrógeno a 77K reflejan una superficie interna prácticamente nula, con lo que la superficie específica de estos sólidos sólo se debe a su superficie externa, la cual, a su vez, no es muy elevada debido al tamaño de las partículas cristalinas formadas. La cristalinidad de las partículas es más evidente en los sólidos que han sido tratados durante $24 \mathrm{~h}$. Sus valores de superficie específica son muy bajos, debido a la formación de partículas más cristalinas y de mayor tamaño que en los sólidos activados durante $6 \mathrm{~h}$.

La microscopía electrónica de barrido es una técnica importante en la caracterización de materiales zeolíticos, ya que permite conocer la forma que adoptan los cristales de las zeolitas (27). Las microfotografías presentadas en la figura 6 correspondientes a los metacaolines tratados en condiciones fuertemente alcalinas, muestran la formación de cristales en forma de cubos (superior) que se encuentran agrupados formando agregados voluminosos cuasi-esféricos (inferior). La comparación entre las microfotografías obtenidas para los distintos sólidos refleja nuevamente la poca influencia que la temperatura de calcinación del caolín y la duración del tratamiento ejercen sobre la cristalización de la zeolita, puesto que todos los sólidos sintetizados muestran cristales con la misma forma.
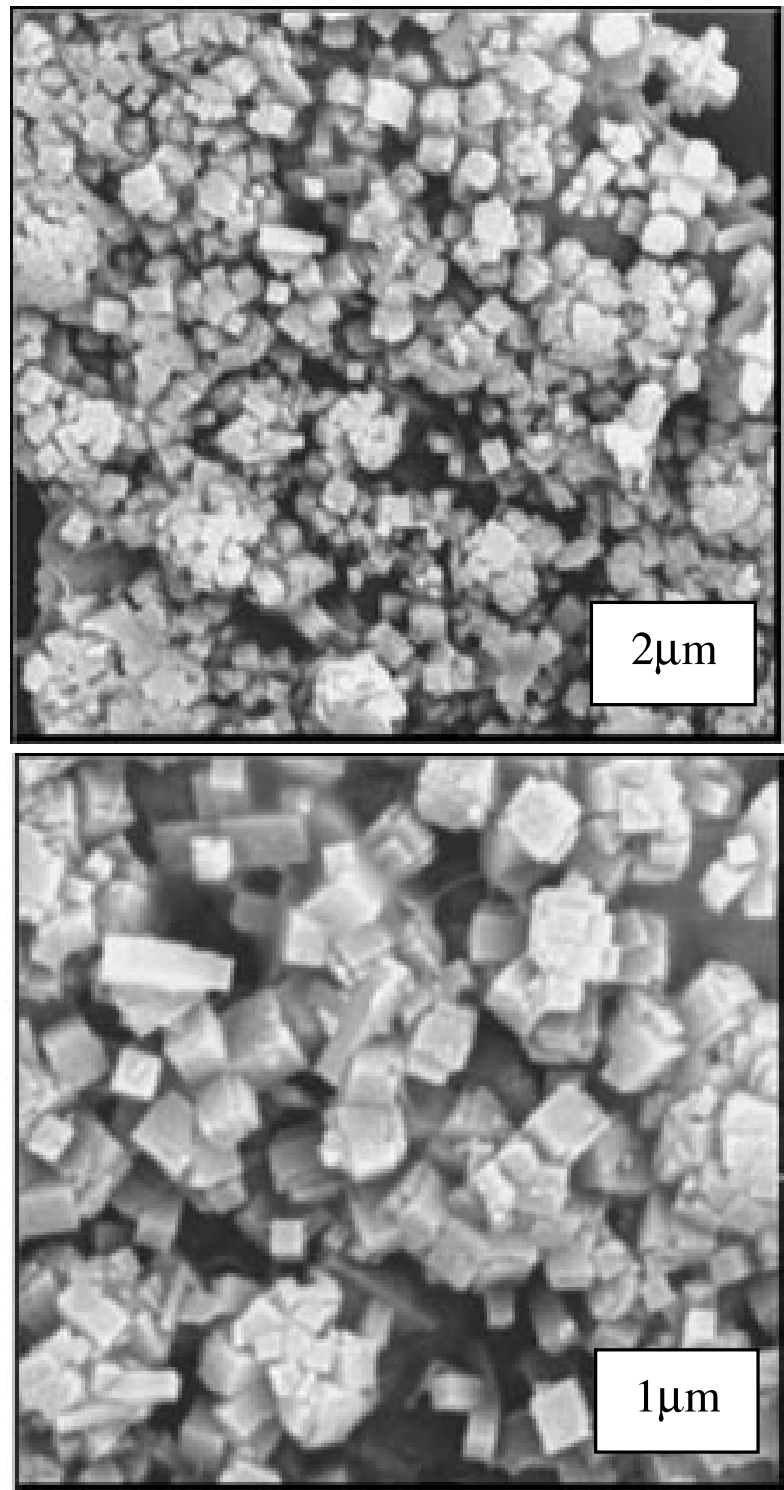

Figura 6. Microfotografías de MEB del MK-600-KOH-24: superior 5000 aumentos, inferior 10000 aumentos. 


\section{CONCLUSIONES}

La calcinación del caolín entre $600-800^{\circ} \mathrm{C}$ da lugar a sólidos amorfos susceptibles de ser modificados mediante tratamientos químicos. La activación ácida de los mismos da lugar a la síntesis de sólidos constituidos por sílice amorfa con elevadas áreas superficiales, 200 $\mathrm{m}^{2} / \mathrm{g}$, y microporosidad, cuando el tratamiento es realizado en condiciones de reflujo durante $6 \mathrm{~h}$. El aumento de la duración del tratamiento ocasiona el colapso de los microporos generados dando lugar a sólidos con superficies específicas inferiores, existiendo entonces unas condiciones idóneas para la generación de sólidos porosos con buenas propiedades texturales. La calcinación del caolín a $900^{\circ} \mathrm{C}$ da lugar a un metacaolín menos reactivo ante el tratamiento ácido, ya que la sinterización de las partículas dificulta el ataque de los protones del ácido.

Por su parte la activación básica se presenta como un método sencillo y eficaz para la síntesis de la zeolita K-F (Z), sin necesidad de llevar a cabo un tratamiento hidrotermal para su obtención. La cristalización de esta zeolita está condicionada por la temperatura empleada y por la composición del material de partida, puesto que ni la duración del tratamiento ni la temperatura de calcinación del caolín producen variaciones significativas en las características de los sólidos sintetizados. A diferencia del tratamiento ácido en este caso los cuatro metacaolines se comportan de forma análoga, no parece existir un metacaolín menos reactivo.

\section{BIBLIOGRAFÍA}

1. J.F. Bartolome. “Caolín: composición, estructura, génesis aplicaciones”. Bol. Soc. Esp. Ceram. V., 36, 7-19 (1997).

2. T. J. Rong, J. K. Xiao. "The catalytic cracking activity of the kaolin-group minerals". Mater. Letters. 57, 297-301 (2002).

3. H.H. Murray, W.D. Keller. "Kaolins, kaolins and kaolin", pp. 1-5 en Kaolin, Genesis and Utilization. Ed. H. Murray, W. Bundy, C. Harvey, The Clay Minerals Society. Boulder, Colorado (EE.UU.) 1993.

4. W.M. Bundy. "The diverse industrial applications of kaolin", pp. 43-73 en Kaolin, Genesis and Utilization. Ed. H. Murray, W. Bundy, C. Harvey, The Clay Minerals Society. Boulder, Colorado (EE.UU.) 1993.

5. F. Bergaya, G. Lagaly. "Surface modification of clay minerals". Appl. Clay Sci. 19, 1-3 (2001).

6. J. Temuujin, G. Burmaa, J. Amgalan, K. Okada, Ts. Jadambaa, K.J.D. Mackenzie. "Preparation of porous silica from mechanically activated kaolinite". J. Porous Mater. 8, 233-238 (2001).

7. R.L. Frost, E. Makó, J. Kristóf, E. Horváth, J.T. Kloprogge. "Mechanochemical treatment of kaolinite". J. Colloid Interface Sci. 239, 456-466 (2001).

8. R.J. Lussier. "A novel clay-based catalytic material. Preparation and properties". J. Catal. 129, 225-237 (1991)
9. S. Chandrasekhar, S. Ramaswamy. "Influence of mineral impurities on the properties of kaolin and its thermally treated products". Appl. Clay Sci. 21, 133-142 (2002).

10. M.A. Vicente, M. Suárez, M.A. Bañares, J.D. López-González. “Comparative FT-IR study of the removal of octahedral cations and structural modifications during acid treatment of several silicates". Spectrochimica Acta Part A 52, 1685-1694 (1996).

11. D.W. Breck. "The synthesis of zeolites from clays", pp. 313-320 en Zeolite Molecular Sieves. Structure, Chemistry and Use. Ed. John Wiley \& Sons. London and New York (1974).

12. E.I. Basaldella, A. Kikot, E. Pereira. “Synthesis of zeolites from mechanically activated kaolin clays". Reactivity of Solids 8, 169-177 (1990).

13. S. Brunauer, P.H. Emmett, E. Teller. “Adsorption of gases in multimolecular layers". J. Am. Chem. Soc. 60, 309-319 (1938).

14. M.M. Dubinin, V.A. Astakhov. "Description of adsorption equilibrium of vapours on zeolites over wide ranges of temperature and pressure". Adv. Chem. Series 102, 69-85 (1971).

15. B.C. Lippens, J.H. De Boer. "Studies on pore systems in catalysis. The $t$ method". J. Catal. 4, 319-323 (1965).

16. R.L. Frost. "Hydroxyl deformation in kaolins". Clays Clay Miner. 46, 280-289 (1998).

17. N.B. Colthup, L.H. Daly, S.E. Wiberley. Introduction to Infrared and Raman Spectroscopy. $3^{\text {rd }}$ Ed. Academic Press. London (1990).

18. J.F. Lambert, W.S. Millman, J.J. Fripiat. "Revisiting kaolinite dehydroxilation: A ${ }^{29} \mathrm{Si}$ and ${ }^{27} \mathrm{Al}$ MAS NMR study". J. Am. Chem. Soc. 111, 3517-3522 (1989).

19. Q. Liu, D.A. Spears, Q. Liu. "MAS NMR study of surface-modified calcined kaolin". Appl. Clay Sci. 19, 89-94 (2001).

20. K. Okada, A. Shimai, T. Takei, S. Hayashi, A. Yasumori, K.J.D. MacKenzie "Preparation of microporous silica from metakaolinite by selective leaching method". Microporous Mesoporous Mater. 21, 289-296 (1998).

21. J.D. Kubicki, G.A. Blake, S.E. Apitz. "Ab initio calculations on aluminosilicate $\mathrm{Q}^{3}$ species: Implications for atomic structures of mineral surfaces and dissolution mechanisms of feldspars". Am. Miner. 81, 789-799 (1996).

22. R.M. Barrer, J.F. Cole, H. Sticher. "Chemistry of soil minerals. Part V. Low temperature hydrotermal transformations of kaolinite". J. Chem. Soc. A, 2475-2485 (1968).

23. W.M. Meier, C. Baerlocher. "Zeolite type frameworks: connectivities, configurations and conformations", pp. 141-159 en Molecular Sieves, Science and Technology. Vol 2. Structures and Structure Determination. Ed. H.G. Karge, J. Weitkamp. Springer, Berlín (1999).

24. D.W. Breck. "Infrared studies of zeolites", pp. 415-425 en Zeolite Molecular Sieves. Structure, Chemistry and Use. Ed. John Wiley \& Sons. Londres y Nueva York, (1974)

25. R.M. Barrer, D.E. Mainwaring. “Chemistry of soil minerals. Part XI. Hydrotermal transformations of metakaolinite in potassium hydroxide". J. Chem. Soc. Dalton Trans., 1254-1259 (1972)

26. F. Rouquerol, J. Rouquerol, K. Sing. Adsorption by powders and porous solids. Principles, methodology and applications. Academic Press. Londres 1999.

27. O. Terasaki. "Electron microscopy studies in molecular sieve science", pp. 72-85 en Molecular Sieves, Science and Technology. Vol. 2. Structures and Structure Determination. Ed. H.G. Karge, J. Weitkamp. Springer, Berlín 1999.

Recibido: 1.2 .03

Aceptado: 30.11 .03 